\title{
Öffentliche Mobilität legitimieren: Das Berliner Mobilitåtsgesetz
}

\author{
Jens Holger Kirchner
}

\section{Einführung: Über allem Stand die Ungeduld oder ,Am Anfang war das Rad“}

Seit vielen Jahren hat in Berlin der Fahrradverkehr zugenommen - absolut und auch relativ. Einige behaupten, wegen der kommunalpolitischen Bemühungen, Andere behaupten, trotz dessen. In einigen Gegenden der Stadt nimmt der Radverkehr schon gefühlt einen Anteil von $50 \%$ auf der Fahrbahn, d. h. gegenüber dem Kfz-Verkehr, ein. Lange Fahrradkolonnen und Pulkbildungen an Kreuzungen in Spitzenzeiten sind das Bild dieser Entwicklung. Selbst Fahrradstaus an der Ampel sind zu beobachten, weil nicht bei jeder Grünphase alle Fahrradfahrenden über die Kreuzung kamen. Fahrradfahren ist in Berlin zweifelsfrei attraktiv geworden - aus gesundheitlichen, Praktikabilitäts-, sicherlich auch ökologischen, mit Sicherheit Lebensstil- und, wie ich beobachten konnte, vor allem aus zeitökonomischen Gründen. Mensch möchte einfach schnell in der Stadt unterwegs sein.

Unstrittig wird auch sein, dass die Diskrepanz zwischen der für einen sicheren Radverkehr vorhandenen Infrastruktur und den tatsächlich im öffentlichen Raum fahrradfahrenden Verkehrsteilnehmenden in Berlin immer größer wurde. Sicherer ist Fahrradfahren in Berlin in den vergangenen Jahren nun leider nicht geworden.

Jens Holger Kirchner war von 2006 bis 2016 Bezirksstadtrat für Öffentliche Ordnung, Verkehr und Stadtentwicklung im Berliner Bezirk Pankow, in den Koalitionsverhandlungen in der AG Verkehr, von 2016 bis 2018 als Staatssekretär für Verkehr in der

Senatsverwaltung für Umwelt, Verkehr und Klimaschutz maßgeblich an der Erarbeitung des Mobilitätsgesetzes beteiligt.

J. H. Kirchner $(\bowtie)$

Der Regierende Bürgermeinster von Berlin, Berlin, Deutschland

E-Mail: jens-holger.kirchner@ senatskanzlei.berlin.de

(C) Der/die Autor(en) 2021 


\section{Die Zeit war reif, aber es begann schon früher}

Es war nicht so, dass zur Förderung des Radverkehrs in Berlin bei Null angefangen werden musste. Bereits im November 2004 wurde die erste Radverkehrsstrategie für Berlin durch den Senat beschlossen. Strategisch fügte sie sich in das Konzept der Verkehrsentwicklungsplanung für eine stadtverträgliche, nachhaltige und sozial gerechte Mobilität ein. Die - wenn auch schleppende Umsetzung der Strategie zeigte ihre Wirkung. Mit der Neufassung des Stadtentwicklungsplans Verkehr im März 2011 wurde über einen modifizierten und erweiterten Maßnahmenkatalog die Radverkehrsstrategie quasi fortgeschrieben. Dennoch öffnete sich die Schere zwischen strategischer Absicht, planerischem Wollen und baulicher Umsetzung - gepaart mit höherem Sicherheitsanspruch und deutlich höherem Verkehrsanteil immer mehr. So ging es nicht weiter.

\section{Volksentscheid Fahrrad: Gesetzentwurf vorgelegt}

In einer Zeit, da gesellschaftliche Konflikte von Bürgerinitiativen und -bewegungen immer lautstarker, polarisierender und emotionaler auf Montagsdemos und Brüllkundgebungen vorgetragen wurden und der gesellschaftliche Diskurs unter immer mehr Vorwurfs- und Unterstellungskultur litt, legte die Initiative für einen Volksentscheid Fahrrad mit einem detailliert ausgearbeiteten Gesetzentwurf einen konstruktiven Vorschlag auf den Tisch und spielte virtuos die Klaviatur der direkten Demokratie (siehe den Beitrag von Schneidemesser in diesem Band). Das muss man erst mal hinbekommen, innerhalb von wenigen Tagen weit über 100.000 gültige Unterschriften zu sammeln. Diese Geschwindigkeit zeigte mehr als deutlich, wie präsent dieses Thema in der Stadt war und wie dringend Lösungen gebraucht wurden. Auch zeitstrategisch passte die Initiative gut in die verkehrspolitischen Zyklen - rechtzeitig vor den nächsten Wahlen, aber nicht zu früh, um wieder in Vergessenheit oder Wiederholungsschleifen zu geraten.

Die damals verantwortliche Politik und Verwaltung reagierten nicht sonderlich souverän. Der Eindruck war nicht ganz zu vermeiden, dass der damalige Verkehrssenat die Initiative eher als Bedrohung ansah, die Forderungen der Initiative als zu weitgehend und forsch eingeschätzt wurden, sich auf die Darstellung des (unstrittig) bisher Erreichten und Begonnenen beschränkt wurde und der Umgang miteinander schließlich eskalierte. Die Kostenschätzungen für die Umsetzung des 
Gesetzes wich beispielsweise deutlich voneinander ab und gab Anlass zu gegenseitigen Unterstellungen und Vermutungen. Von gegenseitiger Wertschätzung, die natürlich Jjede*r vom Anderen zwar einforderte, aber offenbar nicht zu geben bereit war, fehlte jede Spur.

In dieser Gemengelage - Grüne und Linke waren in der Opposition und unterstützten den Volksentscheid Fahrrad - vermischten sich die Rollen. Gerade die Grünen kritisierten lautstark und prononciert die Politik des Verkehrssenators. Dabei vergaßen sie wohl, dass Bürgerinitiativen aus der Stadtgesellschaft heraus entstanden eine andere Rolle haben als eine Partei im Abgeordnetenhaus in der Opposition. Gerade die Grenzüberschreitungen, zu der sich selbst eine Bürgerinitiative nicht hinreißen lassen sollte (auch wenn Provokation und Übertreibung leider zum Handwerkszeug einer heutigen Bürgerinitiative zu gehören scheinen), sind für eine Partei im parlamentarischen Kontext erst recht nicht akzeptabel. Die persönlichen Angriffe durch Vertreter*innen des Volksentscheids Fahrrad auf den damaligen Verkehrssenator Geisel und seinen Staatssekretär Gaebler, aber auch auf führende Vertreterinnen und Vertreter der Verkehrsverwaltung z. B. im Zusammenhang mit tödlichen Verkehrsunfällen wurden mehr oder minder offen unterstützt. Sie mochten mediale Aufmerksamkeit erzeugt haben, führten aber mit Sicherheit zu mehr Konfrontation und Polarisierung - und verbauten mit ebenso großer Sicherheit die Möglichkeit, bei allen unterschiedlichen Auffassungen an einem nur gemeinsam durchzusetzenden Projekt zu arbeiten. Die Stimmung war versaut und die Bereitschaft, aufeinander zuzugehen, tendierte gegen Null. Die Autofahrer*innenlobby und diejenigen, die keine Veränderungen für , mehr Radverkehr' wollten, kamen als Sieger*innen hervor, weil genauso sich eben nichts bewegt hat.

Ungeachtet dessen haben alle Parteien in ihren Wahlprogrammen mehr oder weniger konkrete Aussagen für eine andere Mobilität mit mehr oder weniger konkreten räumlichen Bezügen für Berlin selbst bzw. für die Metropolregion Berlin-Brandenburg gemacht - sicherlich auch ein Ergebnis der öffentlichen Debatte um den Volksentscheid Fahrrad.

\section{$3 \quad$ Koalitionsverhandlungen unter dem Zeichen einer modernen Mobilität}

Nach der Berliner Landtagswahl am 18. September 2016 war relativ schnell klar, dass es zu Koalitionsverhandlungen zwischen der (geschwächten) SPD; der (erstarkten) LINKEN und dem (ebenfalls geschwächten) Bündnis 90/Die Grünen 
kommt. Während die SPD und die LINKE schon mal 10 Jahre zusammen regiert hatten, sie sich also kannten, war für die Grünen die Situation in der neuen Rolle neu und ungewohnt, zumal das Wahlergebnis nach den Prognosen überraschend schwach ausfiel - demokratietheoretisch war das Mandat für eine konsequente, mutige Verkehrswende nun nicht so eindeutig abzuleiten.

Auffällig ist, dass der Verkehrsteil im Koalitionsvertrag ${ }^{1}$ sehr ausführlich und detailliert einzelne Projekte aufführt - sicherlich ein Ausdruck eines nicht gerade vorhandenen Vertrauensverhältnisses unter den künftigen Koalitionspartnern, sicherlich aber auch Ergebnis vorhandener genauer, bereits erarbeiteter und in der Stadtgesellschaft diskutierter Vorstellungen. Das Ringen um Konkretisierung bis hin zu kleinteiligen Festlegungen war Ergebnis einer Sehnsucht, die Verkehrspolitik nun endlich möglichst weit voranzubringen und keine Zeit mehr mit wohlfeilen Absichtserklärungen und unpräzisen Prüfaufträgen zu verlieren. Die guten Vorbereitungen z. B. aus dem Bündnis Pro Straßenbahn mit deren Zielnetzplanungen haben dazu geführt, das zwölf namentlich aufgeführte StraßenbahnProjekte direkt in den Koalitionsvertrag aufgenommen wurden - inklusive Planungs-, Bau- und Fertigstellungszeiten. Konkreter geht es fast nicht.

Für das künftige Mobilitätsgesetz gab es zwei thematische Zugänge, die damals aber noch nicht miteinander verknüpft waren. Einerseits wurde vereinbart, dass das vorhandene ÖPNV-Gesetz zu einem integrierten Mobilitätsgesetz weiterentwickelt wird - wobei ironischerweise im Vertrag selbst ,Mobilitätsgesetz noch in Anführungsstrichen aufgeführt wird, als ob man sich der Ernsthaftigkeit noch nicht so sicher war. Hier wird schon Bezug genommen auf das Ziel, den Fuß- und Radverkehr umfassend neu zu regeln. Aber auch strategische Ziele wie Umweltverbundgedanken, Digitalisierung, Sharing- oder Diversity-Kriterien werden aufgeführt. Auch finanzielle Rahmenfestlegungen, wie die Zweckbindung der Mittel aus dem Entflechtungsgesetz oder dem Gemeindefinanzierungsgesetz, wurden festgehalten.

Es folgten dann in einem eigenen Abschnitt qualitative Zielorientierungen für den Fußverkehr - ohne einen Zusammenhang zum Mobilitätsgesetz herzustellen.

In einem weiteren umfangreichen Abschnitt wurde dann allerdings - mit Sicherheit der vorangegangenen Diskussion in der Stadt um das Radgesetz geschuldet - unter der Überschrift „Neue Impulse für einen großstadtgerechten Radverkehr" dezidiert aufgeführt, wie die Koalition in einem ersten Schritt bis zum Frühjahr 2017 (sic! das war im Dezember 2016 und nicht nur sehr

\footnotetext{
${ }^{1}$ https://www.berlin.de/rbmskzl/regierender-buergermeister/senat/koalitionsvereinbarung/
} 
ambitioniert, sondern auch völlig in Ignoranz von sorgfältigen Gesetzgebungsprozessen in einem demokratischen Gemeinwesen, wie sich schnell herausstellte) einen Gesetzentwurf für den Radverkehr vorlegen und einbringen wird, wie der Dialog mit der Stadtgesellschaft dazu geführt werden soll und welche Rahmenbedingungen zum Ausbau der Fahrradinfrastruktur geschaffen werden müssen:

1. ein Bündnis für den Radverkehr

2. die InfraVelo $\mathrm{GmbH}$, ein städtisches Unternehmen als Koordinierungsstelle Radverkehr

3. Mindestbereitstellung zusätzlicher Personal- und finanzieller Ressourcen für die Umsetzung auf Landes- und Kommunalebene (Bezirk)

4. zusätzlich 40 Mio. EUR für 2018 und ab 2019 dann 50 Mio. EUR ausschließlich für die Radinfrastruktur

Darüber hinaus wird in einem weiteren Abschnitt genau aufgeführt, welche Bausteine für eine sichere Radinfrastruktur vorzusehen sind (u. a. Radwegenetze, sichere Kreuzungen, physische Trennung des Radverkehrs vom übrigen Verkehr). Diese Präzisierungen in den Vereinbarungen sollten sich später auszahlen, da innerhalb der Fraktionen darauf Bezug nehmend Verbindlichkeit eingefordert werden konnte. Umgekehrt war es aber umso schwieriger, einzelne Punkte durchzusetzen, die nicht im Koalitionsvertrag festgelegt waren.

Anfang Dezember 2016 wurde der Koalitionsvertrag unterzeichnet und die neue Regierung konstituierte sich. Schnell wurde in der neu gebildeten Senatsverwaltung für Umwelt, Verkehr und Klimaschutz (SenUVK) die Umsetzung des Koalitionsvertrags auf die Agenda gesetzt und die organisatorischen und strategischen Weichen gestellt. Dazu musste - neben der Neuorganisation des Hauses - auch formal entschieden werden, wer an der Verhandlungsgruppe teilnimmt und wie die Prozesse der Erarbeitung und der Beteiligung/Rückkopplung in die Stadtgesellschaft umgesetzt werden. Es lag ein enormer Erwartungsdruck auf der nunmehr von den Grünen geführten Verkehrs-Senatsverwaltung. Die Grünen mussten plötzlich liefern und ihre eigenen (unrealistischen) zeitlichen Forderungen erfüllen. Das lief auch parteiintern nicht ohne Verwerfungen ab.

Die Festlegungen aus dem Koalitionsvertrag konnten sinnvoller Weise nur durch eine Verknüpfung erfüllt werden. Das Konstrukt des künftigen Mobilitätsgesetzes nach dem Bild eines griechischen Tempelportals (siehe Abb. 1) drängte sich quasi von selbst auf: ein allgemeiner Teil für Zielformulierungen einer nachhaltigen, sicheren, sozialen, barrierefreien, stadtverträglichen, ökologischen (wahlweise klimaneutralen) und menschengerechten Mobilität in der Metropolregion, Begriffsbestimmungen und die Beschreibung der Planungsinstrumente 


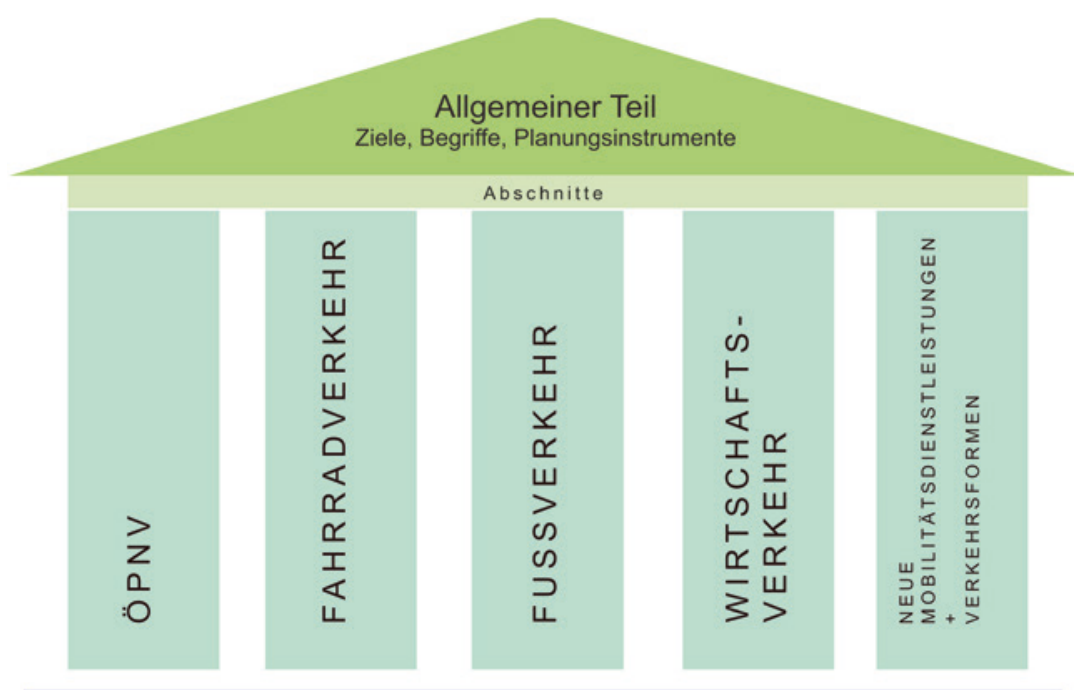

Planwerke, Verordnungen, Ausführungsvorschriften, Regelwerke

Abb. 1 Die fünf Säulen des Berliner Mobilitätsgesetz. (Quelle: Eigene. Darstellung)

als Rahmen und Überbau. Daraus abgeleitet die fünf tragenden Säulen als Abschnitte für den ÖPNV, den Fahrradverkehr, den Fußverkehr, den Wirtschaftsverkehr und nicht zuletzt für Neue Mobilitätsdienstleistungen und Verkehrsformen (wobei letzterer Teil alleine durch die fortlaufenden Überlegungen und bundesweit geführten Debatten um eine Antriebswende, autonomes Fahren, vernetzte Mobilitätsdienstleistungen usw. mehrfach den Namen änderte). Als Basis für dieses Gesetzeswerk sind in diesem Konstrukt die Planwerke, Verordnungen, Ausführungsvorschriften, Regelwerke und sonstigen Bestimmungen zu sehen, deren solide Fundamentierung es zwangsläufig für die Umsetzung bedarf, um das Gebäude dauerhaft rechtlich standsicher zu machen und nicht zum Einsturz zu bringen.

Unstrittig, wenn auch nicht ohne Kritik, wurden in dieser Gemengelage gesetzliche Regelungen für den ÖPNV wegen der auslaufenden Befristung des bestehenden ÖPNV-Gesetzes und gesetzliche Regelungen für den Radverkehrsteil wegen dem enormen öffentlichen Erwartungsdruck zuerst erarbeitet - ergänzt durch den allgemeinen Teil. Die gesetzlichen Regelungen für den Fußverkehr, den Wirtschaftsverkehr und die neuen Mobilitätsdienstleistungen 
und Verkehrsformen sollten dann in den Folgejahren das Gesetz ergänzen - aus Kapazitätsgründen, aber auch, weil damals noch das Integrierte Wirtschaftsverkehrskonzept in Erarbeitung war und gesetzlicher Regelungsbedarf erst nach Vorliegen desselben abgeleitet werden sollte. Von verschiedenen Interessenseiten wurde im gesamten Gesetzgebungsprozess gefordert, auch einen Abschnitt zum motorisierten Individualverkehr einzufügen. Dem wurde nicht entsprochen. Zum einen, weil Aspekte der privaten Pkw-Nutzung Gegenstand in dem kommenden Baustein zu neuen Mobilitätsdienstleistungen und Verkehrsformen (beispielsweise Carsharing oder autonomes Fahren) behandelt werden. Zum anderen, weil im Abschnitt Wirtschaftsverkehr unternehmerische Aspekte z. B. des Lieferverkehrs berücksichtigt werden. Zudem ist der Autoverkehr umfassend in der Straßenverkehrsordnung, im Berliner Straßengesetz und in bundesweit geltenden Richtlinien geregelt. Es wurde immer wieder daran erinnert, dass das Mobilitätsgesetz u. a. ein neues Gleichgewicht zwischen den Verkehrsträgern schaffen soll und für den Radverkehr und den ÖPNV gesetzlich das regelt, was für den Automobilverkehr schon lange selbstverständlich ist.

Bereits im Februar 2017 konstituierte sich die Verhandlungsgruppe auf Einladung der Senatorin für Verkehr, durchgängig geleitet und moderiert vom damaligen Staatssekretär für Verkehr und organisatorisch und redaktionell begleitet von Kolleg*innen des Center Nahverkehr als externem Dienstleister. Teilnehmende dieser Verhandlungsgruppe waren neben Vertreter*innen der Verkehrsabteilung von SenUVK und der Senatskanzlei Vertreter*innen vom Volksentscheid Fahrrad, vom Berliner ADFC und vom BUND-Landesverband Berlin. Vom Abgeordnetenhaus waren jeweils zwei Vertreter*innen der Regierungsfraktionen SPD, Die Linke und Bündnis 90/Die Grünen in der Verhandlungsgruppe. Die Verhandlungsgruppe tagte zwischen März und Juni insgesamt zwölfmal, teilweise im Wochentakt und bis tief in die Nacht. Es wurde verabredet, Leitlinien/Eckpunkte auf der Basis des vom Volksentscheid Fahrrad vorgelegten Gesetzvorschlags zu erarbeiten und darauf aufbauend den Gesetzentwurf $\mathrm{zu}$ erarbeiten. Ausgesprochen hilfreich war die Installation von verschiedenen Arbeitsgruppen in kleinerer Besetzung, die jeweils zur nächsten Sitzung der Verhandlungsgruppe Vorschläge erarbeitet haben.

Wie ein roter Faden zog sich durch die Verhandlungen in der Gruppe das fehlende Vertrauen der Initiativen und Verbände gegenüber der Verwaltung und Teilen der Politik (wenn auch unterschiedlich ausgeprägt) - gepaart mit einer ausgeprägten Unkenntnis über Planungs-, Bau- und Verwaltungsverläufe, aber auch mit einer wenig ausgeprägten Bereitschaft, bundesgesetzliche Regelungen anzuerkennen. Die nicht sehr ausgeprägte Bereitschaft der Verwaltung, die Verbände und Initiative als Partner auf Augenhöhe zu akzeptieren wurde ergänzt durch eine 
Fremdheit gegenüber der Diskussionskultur und dem Kommunikationsstil der Initiative und den Verbänden. Auch das fehlende Vertrauen der Koalitionspartner untereinander gepaart mit dem Überprüfen eines jeden Vorschlags, ob dieser nur geäußert wurde, um dem Adressaten oder der Adressatin zu schaden, ergänzte die Atmosphäre. Vor diesem Hintergrund war es mitunter mehr als einmal nötig, die Verhandlungsgruppe an das gemeinsame Ziel zu erinnern, professionelle Distanz und Konstruktivität einzufordern und dafür zu werben, dass jede*r Teilnehmende nicht nur inhaltlich Verantwortung zum Gelingen der Gesetzesinitiative beiträgt, sondern auch zur Einhaltung des vorgegebenen Zeithorizonts. Dieser Widerspruch blieb bis zuletzt eminent prägend - lautstark die wiederholten Verzögerungen kritisieren, aber stundenlang libidinös eine Angelegenheit diskutieren, wobei die Gleichung ,Bedeutung des Details im umgekehrt proportionalen Verhältnis zur Behandlungsdauer' oft zutraf.

Die gesamte Gesetzesinitiative - nicht nur die Phase der Beratungen in der Verhandlungsgruppe - standen unter dem Druck der im Koalitionsvertrag verankerten zeitlichen Vorgaben. Diesem Druck standzuhalten und solide Arbeit abzuliefern - auch wenn es länger dauert als vorgestellt - war nicht einfach. Und es sollte sich herausstellen, dass die Erarbeitung eines solchen Gesetzes ohne anwendbares Vorbild, ohne inhaltliche Blaupause, aber auch ohne Verfahrensblaupause, inklusive einer breiten Beteiligung der Stadtgesellschaft und inklusive der seriösen Behandlung durch die Verfassungsorgane des Landes Berlin eben seine Zeit braucht und von Mitte Februar 2017 bis Mitte Juli 2018 - nüchtern betrachtet - in einem Zeitraum von nicht mal 1,5 Jahren ausgesprochen intensiv und schnell erfolgte. Schneller ging es nicht und wenn es schneller gegangen wäre, wäre das Verfahren oder gar das Gesetz selbst angreifbar gewesen. Es war immer wieder feststellbar, dass aus offensichtlicher Unkenntnis über Fristen, Verläufe und zwingend zu Beteiligende die Gleichung aufgemacht wurde: Leitlinien fertig, Gesetzentwurf kein Problem, Beschluss morgen, Umsetzung auf der Straße ab heute. So mussten teilweise die Beteiligten daran erinnert werden, dass es ein Gebot der Sorgfalt und der Rechtsfolgeprüfung einzuhalten gilt, dass es Verbändebeteiligung, Mitzeichnungserfordernisse, einen Rat der Bürgermeister*innen gibt sowie eine gesetzgebende Versammlung - Berliner Abgeordnetenhaus genannt.

Interessanterweise wurde ab Mitte 2017, also schon ein Jahr zuvor, vielfach in der Stadt gefordert, dass doch die sich abzeichnenden Regelungen aus dem Mobilitätsgesetz anzuwenden sind. Dieser faktische Vorgriff einer normativen Kraft zeigte auch, wie dringend diese gesetzlichen Regelungen erwartet wurden und welch hohen Stellenwert das Gesetz schon hatte, bevor es überhaupt in Kraft getreten war. 
Fachlich war eine der Hauptaufgaben der Verhandlungsgruppe, die vielfältigen, fast unüberschaubaren, teilweise sehr detaillierten Vorschläge und Vorstellungen daraufhin zu sortieren, ob sie im Gesetz verankert sein müssen bzw. können, im Radverkehrsplan oder anderen Planwerken geregelt werden können, oder in Verordnungen bzw. Ausführungsvorschriften aufgenommen werden sollten - oder ob es einer Bundesratsinitiative bedarf, um z. B. die StVO zu ändern. Hierfür brauchte es einige Zeit, um alle am Tisch davon zu überzeugen. Um jeden Zentimeter, jede konkrete qualitative, insbesondere aber auch jede quantitative Vorgabe wurde leidenschaftlich gestritten. Gerade hier wurden die sehr unterschiedlichen Vorstellungen von verbindlichen Vorgaben (10 sichere Kreuzungen im Jahr, in den Folgejahren pro Jahr 20-30 umbauen, Mindestbreite von Radverkehrsanlagen von $2 \mathrm{~m}$ in einem gebauten städtischen Umfeld, $100 \mathrm{~km}$ (geschützte) Radwege im Jahr bauen, 100.000 Fahrradbügel aufstellen usw. usf.) in einem Gesetz verankert - deutlich und nur schwer vereinbar. Auch über bestimmte Definitionen - z. B. über ein Vorrangnetz für den Radverkehr - wurde intensiv und ausdauernd gestritten - teilweise auch aneinander vorbeigeredet, weil die einen darunter ein Netz von Verkehrswegen mit Vorrang für den Radverkehr verstanden, andere eher ein Netz von Verkehrswegen, welches für den Radverkehr vorrangig umzubauen ist, weil es derzeit in besonders schlechtem Zustand bzw. besonders unsicher ist. Das kostete Zeit.

Andere Punkte wie die Verankerung der Vision Zero als strategisch-normatives Ziel, das Verfahren nach besonders schweren Unfällen, Strukturen wie die gesetzliche Verankerung des FahrRates als beratendes Gremium, einer Koordinierungsstelle Radverkehr oder des Bündnisses für Radverkehr, aber auch solch praktische Angelegenheiten wie die Berechtigung der BVG selbst auf zugeparkten Busspuren abschleppen zu dürfen, waren dagegen schnell unstrittig. Und es war ein Lernprozess für die gesamte Verhandlungsgruppe, aus dem durch die Vorgeschichte stark vorgeprägten Fokus auf den Radverkehr ein Gesetz für alle Verkehrsteilnehmenden zu machen, worin der Radverkehr ,nur' noch als ein Abschnitt vorkommt.

Wiederum andere Punkte, wie die Verankerung auch einer individuell gefühlten Verkehrssicherheit als planerisches Ziel waren inhaltlich unstrittig, allerdings wie der allseits unterstütze formulierte Prüfanspruch an jede planerische Maßnahme, konzentriert auf die Frage: ,Würden Sie hier Ihr achtjähriges Kind alleine fahren/laufen lassen?` in ein Gesetz verankern kann, blieb lange unklar. Deutlich wurde bei diesem Themenkomplex, dass die besten technischen und räumlichen Verkehrssicherheitsstandards nicht viel taugen, wenn die Verkehrsteilnehmenden sich trotzdem unsicher fühlen. 


\section{$4 \quad$ Beteiligung macht besser}

Die Leitlinien wurden im eigens für die Erarbeitung des Mobilitätsgesetzes erweiterten Mobilitätsbeirat vorgestellt und diskutiert. Der Beirat besteht schon seit Jahren und berät in seiner sehr breiten Zusammensetzung (Mobilitätsverbände, BVG, IHK, Polizei, verschiedene Senatsverwaltungen, einige Bezirke, Fraktionen des Abgeordnetenhauses ...) die Senatsverwaltung für Verkehr z. B. bei der Erarbeitung des Stadtentwicklungsplanes Mobilität und Verkehr StEPMoVe. Da lag es nahe, durch eine Erweiterung mit Behinderten- und Seniorenverbänden dieses Format für eine intensive Kommunikation, eine präzise Information und breite Beteiligung zu nutzen.

Danach wurden die ersten Bausteine für einen Referentenentwurf formuliert, wobei dieser Prozess nicht linear verlief, sondern die einzelnen Abschnitte und Paragraphen fragmentarisch zusammengestellt wurden, ohne die Querbezüge und Zusammenhänge aus den Augen zu verlieren. Der sog. Referentenentwurf wurde in einer kleineren Gruppe redaktionell fertiggestellt und nach ,Freigabe der Verhandlungsgruppe in eine informelle Beteiligungsrunde eingespeist, in der verschiedenen Senatsverwaltungen um Stellungnahme und Hinweise gebeten wurden. Auch wurde der Referentenentwurf in seinen Grundzügen nochmals im Mobilitätsbeirat vorgestellt und diskutiert. Ebenfalls wurde eine (vorgezogene) Verbändebeteiligung durchgeführt. Die daraus resultierenden zahlreichen (knapp 800) Stellungnahmen und Hinweise aus allen fach- und parteipolitischen Himmelsrichtungen wurden in diversen Einzelgesprächen mit den Einreichenden erörtert, übernommen, modifiziert, abgewogen. Das Gesetz ist dadurch besser sortiert, diskriminierungsfreier, präziser in den Formulierungen und praxisorientierter für die Anwendung geworden. Die Regelung, bei schweren Unfällen mit Radfahrenden ein Verfahren zur Beseitigung der Unfallursachen durchzuführen, ist z. B. verallgemeinert worden für alle Verkehrsteilnehmende und in den allgemeinen Teil verschoben worden.

Nach einer erneuten Überarbeitung und Abwägung der eingegangenen Hinweise mit einer angepassten Neuordnung der inneren Logik konnte der Gesetzentwurf vor Einbringung in den Senat in das offizielle Mitzeichnungsverfahren gebracht werden. Bei diesem werden alle Senatsverwaltungen um Mitzeichnung gebeten, was mit oder ohne Hinweise erfolgt. Parallel dazu fanden wiederum diverse Einzelgespräche mit den involvierten Behördenabteilungen statt, um die Belange aus diesen Häusern möglichst konfliktfrei aufzunehmen oder anderweitige Verabredungen zu vereinbaren (z. B. eine parallele Bundesratsinitiative für höhere Bußgelder). 
Da die meisten Punkte schon vorab geklärt waren, konnte die Vorbereitung der Senatsbefassung inklusive eines Chefgespräches zwischen dem Regierenden Bürgermeister und der Senatorin zur abschließenden Entscheidung über einige wenige strittige Punkte (z. B. die Verankerung des Verbandsklagerechtes oder die BVG-Ermächtigung zum Räumen zugeparkter Busspuren) sehr schnell - innerhalb von 2-3 Wochen - erfolgen, sodass die 1. Befassung im Senat zwar mediale Aufmerksamkeit erzeugte, aber de facto ,nur' noch eine Formsache war.

Durch die Einbringung in den Rat der Bürgermeister*innen wurde im Gesetzgebungsverfahren die nächste Stufe eingeleitet. Die Berliner Bezirke haben sich bei der Behandlung des Gesetzentwurfes (leider oder zum Glück?) nicht zu einer gemeinsamen Stellungnahme durchringen können, sodass es aus jedem Bezirk teils umfangreiche, aber auch sich widersprechende Stellungnahmen gegeben hat. Auch hier folgten wiederum diverse Einzelgespräche mit den Bezirken, um deren Hinweise zu verstehen, zu erörtern und ggf. mit in den Gesetzentwurf aufzunehmen. In einer 2. Lesung im Rat der Bürgermeister*innen wurde die nun nochmals geänderte Fassung des Gesetzentwurfs vorgestellt und über die übernommenen und abgelehnten Änderungen informiert.

Diese angepasste Fassung des Gesetzentwurfs ist dann in der 2. Lesung vom Senat beschlossen und an das Abgeordnetenhaus zur Behandlung und Beschlussfassung übergeben worden. Trotz allen zeitlichen Drucks, der ja auch aus den Reihen des Parlaments selbst kam, nahm sich das Parlament durchaus Zeit, um in zwei Ausschuss-Anhörungen (Verkehr und Wirtschaft) den Vertretenden der verschiedenen ,Lager" noch einmal die Möglichkeit zu geben, ihre Positionen darzulegen. Alle Angehörten würdigten den bis dahin ausgesprochen intensiven Beteiligungsprozess, auch wenn in der Sache den (bekannten) Positionen nichts hinzugefügt wurde. Auch in der dann folgenden Debatte in der jeweils nächsten Ausschuss-Sitzung wurden die bis dato bekannten Positionen der Regierungsund Oppositionsparteien dargelegt, die eingereichten Änderungsanträge der Regierungsfraktion angenommen und die Änderungsanträge der Opposition allesamt abgelehnt. Ein wirklicher Austausch oder gar eine konstruktive Debatte fand nicht mehr statt, was auch sicherlich einiger Anträge der AfD geschuldet war, die z. B. mit einem der Änderungsanträge die gesamte Streichung des ÖPNVAbschnitts vorschlugen, weil es ja ein ÖPNV-Gesetz gäbe.

Der Gesetzentwurf wurde dann in einer weiteren Sitzung des Hauptausschusses behandelt, ehe er am 28. Juni 2018 in der 2. Lesung in der Plenarsitzung des Abgeordnetenhauses mit großer Debatte, in der die bekannten Positionen noch mal öffentlich kundgetan wurden, erneut erörtert und dann mit den Stimmen der Koalitionsfraktionen gegen die Stimmen der Oppositionsfraktionen mehrheitlich beschlossen wurde. Dem voraus gingen eher diskrete Verhandlungen 
zwischen einigen wenigen Vertretenden der Regierungskoalition, in der die bis dahin noch strittigen Punkte abschließend besprochen und entschieden wurden: Eine Kompromissformel für die angemessene Berücksichtigung des Autoverkehrs in der Präambel, die Verankerung des Verbandsklagerecht blieb weiterhin außen vor. Dafür kam die BVG-Ermächtigung für das Abschleppen von Falschparkenden auf Bus-Spuren wieder rein.

Für die ganz Ungeduldigen schien nunmehr der Gesetzgebungsprozess abgeschlossen zu sein. Dennoch dauerte es nochmals etliche Wochen, bis das Gesetz in Kraft trat. Es wurde am 5. Juli ausgefertigt, am 9. Juli verkündet und trat am 17. Juli 2018 dann endlich mit der Veröffentlichung im Amtsblatt als „Gesetz zur Neuregelung gesetzlicher Vorschriften zur Mobilitätsgewährleistung“ (so etwas können nur Juristen formulieren) in Kraft.

\section{$5 \quad$ Blick zurück nach vorn}

In der Rückschau, nunmehr zwei/drei Jahre später, war die gesamte Entwicklung von der Initiative für einen Volksentscheid Fahrrad über den vorgelegten Gesetzentwurf bis zu den Koalitionsverhandlungen und den Gesetzgebungsprozess für ein Berliner Mobilitätsgesetz ein Musterbeispiel für lebendige, konstruktiv gestaltete demokratische Aushandlungsprozesse. Das schließt Streit ausdrücklich mit ein und ist ein Beweis für die gesellschaftsverändernde Kraft, die demokratisch geführte Diskurse entfalten können. Immer wenn es gelingt, sich auf ein gemeinsames Ziel zu verständigen, einen halbwegs anständigen Umgang miteinander zu pflegen, Abwägung zuzulassen und in die Stadtgesellschaft transparent zu kommunizieren, können nicht nur parlamentarische Mehrheiten entstehen, sondern sich im besten Fall auch die realen Bedingungen in der Stadt ändern - selbst und vielleicht auch nicht ohne Grund gerade in Berlin.

Gesetzgebungsverfahren mit solch einer Beteiligungstiefe kosten Zeit, Kraft und Ressourcen. Welten treffen aufeinander. Dabei kommt der Erarbeitung einer vertrauensvollen Atmosphäre große Bedeutung zu - zeitlich, kräftemäßig und organisatorisch. Es gelang, die anfängliche Konfusion in eine Kernfusion umzuwandeln. Somit wurde viel Energie frei und hat ein Gesetz erschaffen, was es so noch nicht gab. Die Atmosphäre ist stets fragil gewesen und bedurfte besonderer Aufmerksamkeit. Verhandlungen bis zur Erschöpfung, emotionale Ausrutscher, Missverständnisse, Vertrauensbrüche, ,klärende Gewitter ‘ aber auch geniale Momente begleiteten den Verhandlungs- und Gesetzgebungsprozess und sind vom Aufwand und ihrer Bedeutung für das Gelingen eines solchen Unterfangens nicht zu unterschätzen - Beziehungsarbeit pur. 
Dabei haben alle Beteiligten einen Wandlungsprozess durchschritten - vor allem in den jeweiligen Rollenverständnissen. Oppositionsparteien waren auf einmal in der Regierungsverantwortung und mussten plötzlich (in Erfüllung ihrer eigenen Forderungen) liefern oder ihre Regierungspositionen rechtfertigen. Die Initiative war plötzlich Teil des Systems und musste den gemeinsam erarbeiteten Entwurf gegenüber einer kritischen Öffentlichkeit verteidigen. Ihre eigenen Vorstellungen von Transparenz und Öffentlichkeit führten zu einem Enthüllungsaktivismus und führten in eine schwere Vertrauenskrise, die zeitweise den gesamten Gesetzgebungsprozess infrage stellte. Und die Verwaltung sah sich oft in dem Zwiespalt, viele Regelungen des Gesetzes zwar inhaltlich mitzutragen oder für gut zu befinden, aber die vielen Umsetzungskonkretionen mit dem Wissen um die bisherigen knappen Ressourcen, aber auch um die Wirkungsmacht der bundesrechtlichen Straßenverkehrsordnung eher für überzogen einschätzen zu müssen.

\section{Fazit: Und die Ungeduld bleibt notwendig}

Für die Einschätzung, was ein solches Gesetz nun in Praxis bewirkt, ist es fast noch zu früh. Sämtliche laufenden Straßenbauplanungen wurden überarbeitet. Die im Koalitionsvertrag vereinbarten 50 Mio. EUR jährlich aus dem Koalitionsvertrag wurden zur Verfügung gestellt und können gar nicht so schnell umgesetzt werden. Einiges ist begonnen worden, wie die farbliche Markierung vorhandener Radwege oder die Planung der Radschnellwege durch die neu gegründete InfraVelo GmbH. Die Konstituierung und Besetzung der Koordinierungsstelle Radverkehr ist erfolgt. Etliche Bausteine in den Umsetzungsstrukturen sind noch offen, konnten noch nicht oder nur teilweise umgesetzt werden. Die bezirklichen Radverkehrsplanenden-Stellen sind noch immer nicht in allen Bezirken komplett besetzt. Einige Bezirke haben Fahrradstraßen-Netzpläne entwickelt. Der Radverkehrsplan, die Netzplanungen, die Regelpläne sind noch nicht fertig. Auch das Bündnis für Radverkehr als das Koordinierungs- und Arbeitsgremium zur Beschleunigung der Baumaßnahmen selbst steckt noch in den Anfängen. Immerhin ist der Abschnitt Fußverkehr jetzt ins Verfahren gegangen. Noch offen sind die beiden anderen Abschnitte. Das Gesetz hat den Rahmen geschaffen. Die Umsetzung in der gesamten Metropolregion, und das dürfte jeder/m klar sein, wird nicht in eineinhalb Jahren erfolgen können. Aber etwas mehr Ungeduld und Tempo braucht es schon. Das Gesetz allein reicht dazu nicht. 
Open Access Dieses Kapitel wird unter der Creative Commons Namensnennung 4.0 International Lizenz (http://creativecommons.org/licenses/by/4.0/deed.de) veröffentlicht, welche die Nutzung, Vervielfältigung, Bearbeitung, Verbreitung und Wiedergabe in jeglichem Medium und Format erlaubt, sofern Sie den/die ursprünglichen Autor(en) und die Quelle ordnungsgemäß nennen, einen Link zur Creative Commons Lizenz beifügen und angeben, ob Änderungen vorgenommen wurden.

Die in diesem Kapitel enthaltenen Bilder und sonstiges Drittmaterial unterliegen ebenfalls der genannten Creative Commons Lizenz, sofern sich aus der Abbildungslegende nichts anderes ergibt. Sofern das betreffende Material nicht unter der genannten Creative Commons Lizenz steht und die betreffende Handlung nicht nach gesetzlichen Vorschriften erlaubt ist, ist für die oben aufgeführten Weiterverwendungen des Materials die Einwilligung des jeweiligen Rechteinhabers einzuholen.

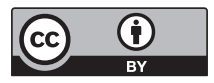

